\title{
A Low-Cost Multi-Modal Auditory-Visual-Tactile Framework for Remote Touch
}

\author{
Filippo Sanfilippo \\ Dept. of Engineering Sciences \\ University of Agder (UiA) \\ Jon Lilletuns vei 9, 4879, Grimstad, Norway \\ Dept. of Mech., Electronic and Chemical Eng. \\ Oslo Metropolitan University (OsloMet) \\ PO box 4 St. Olavs plass, 0130, Oslo, Norway \\ filippo.sanfilippo@uia.no
}

\author{
Claudio Pacchierotti \\ CNRS, Univ Rennes, Inria, IRISA \\ Campus Universitaire de Beaulieu, 35042 Rennes, France
}

\begin{abstract}
Haptic technology for human augmentation provides gains in ability for different applications, whether the aim is to enhance "disabilities" to "abilities", or "abilities" to "super-abilities". Commercially-available devices are generally expensive and tailored to specific applications and hardware. To give researchers a haptic feedback system that is economical, customisable, and fast to fabricate, our group developed a low-cost immersive haptic, audio, and visual experience built by using off-the-shelf (COTS) components. It is composed of a vibrotactile glove, a Leap Motion sensor, and an head-mounted display, integrated together to provide compelling immersive sensations. This paper proposes a higher technology readiness level (TRL) for the system to make it modular and reliable. To demonstrate its potential, we present two human subject studies in Virtual Reality. They evaluate the capability of the system in providing (i) guidance during simulated drone operations, and (ii) contact haptic feedback during virtual objects interaction. Results prove that the proposed haptic-enabled framework improves the performance and illusion of presence.

Index Terms - Wearable Haptics, Human-Computer Interaction, Multimodality.
\end{abstract}

\section{INTRODUCTION}

Multimodal human-computer interaction (MMHCI) refers to the "interaction with the virtual and physical environment through natural modes of communication" [1]. The main objective of MMHCI is to enable a more free and natural communication by providing a transparent bidirectional interface between users and automated systems. Historically, this intercommunication is typically based on one-directional channels of information. To pass human commands to machines, keyboard, mouse and joystick inputs are usually used from one side. Flipside, visual and audio information is frequently sent back from the automated systems to the user. There is no kinesthetic energy flow to the operator in this information loop because there is no sense of touch involved.

Yet touch is one of the most accurate and strong senses, and it is important to our memory and discernment. To exploit the sense of touch and therefore provide the user with additional and intuitive knowledge, it is possible to use haptic technology [2], [3]. Haptic feedback, also known as haptics, is the adoption of the sense of touch in a human-computer interface. With the use of haptics,

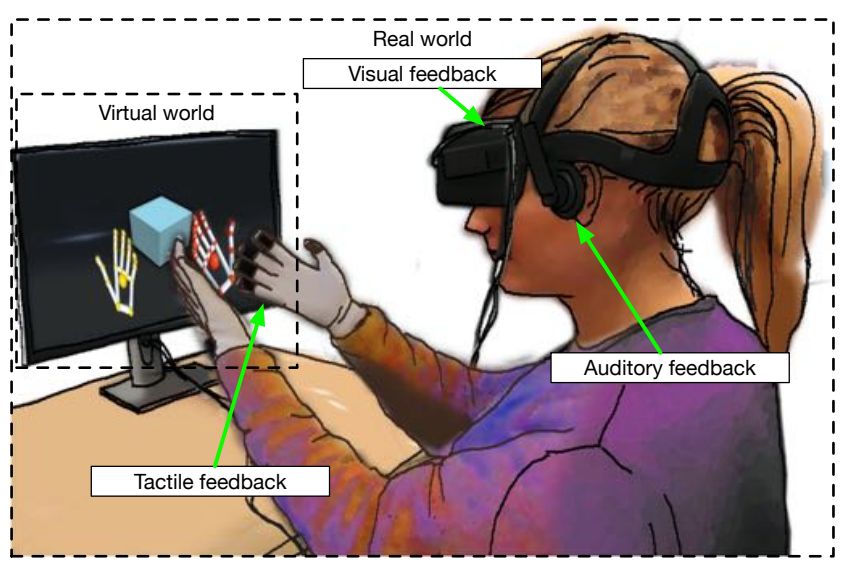

Fig. 1: Proposed idea: develop a low-cost multi-modal auditory-visual-tactile framework for remote touch.

a range of applications are made possible, including the expansion of human abilities: increasing physical strength, improving manual dexterity, augmenting the senses, and most fascinating, projecting human users into remote or abstract environments.

Currently, there are various commercial off-the-shelf (COTS) haptic feedback devices [4], [5]. Nonetheless, most of the currently available devices are still quite costly. In addition, most of these systems are based on proprietary hardware and software. While various haptic software libraries are available, these tools still require significant programming skills to be integrated [6]. This process can therefore often be a frustrating and time-consuming activity, which can cause researchers to concentrate too much on language and syntax, preventing them from paying adequate attention to the logic of their applications. Furthermore, most of the currently available libraries do not provide versatile methods for modelling, simulation and analysis. Scientists are therefore forced to develop custom solutions that often suffer from a lack of generality. More notably, with currently available COTS tools, it is still realtively difficult to fully address the realization of a combined and truly interactive haptic, audio and visual experience. 
To allow researchers to incorporate haptic capabilities in a more versatile, interactive and transparent manner to their applications, our research group has previously proposed a low-cost platform for a fully immersive haptic, audio and visual experience [7]. This system allows haptic features to be easily integrated to various applications. This is made possible by employing vibration actuators and open-source electronics in a newly-designed, low-cost pair of haptic gloves. Hand and finger movements are tracked with a Leap Motion sensor [8] and intuitive visual stereoscopic feedback is received with a Oculus Rift headmounted 3D display [9]. An additional bidirectional audio channel is provided by using an headset with a built-in microphone. To successfully incorporate these elements, the Unity cross-platform 3D-environment [10] is chosen. The proposed architecture enables the creation of a truly immersive user experience. It is possible to create a realtime one-to-one communication between the real world and the virtual world therefore enabling a range of applications from industry and science.

In this work, we aim at improving the robustness of the prototypes designed with the framework. For this reason, the concept of modularity is stressed even more than before concerning the control architecture. Providing an effective multi-modal experience is the main scope of the new framework. The underlying idea is shown in Fig. 1. The usability of the framework is studied and evaluated. The potential of the proposed framework is demonstrated by presenting two human subject studies as experiments of virtual interaction. In the first case study, the user wearing the gloves controls a quadcopter drone model while navigating a scenario cluttered with obstacles. In the second case study, the user interacts with a virtual object by using the index finger. The corresponding path tracking for the fingertip is considered by comparing the user performance with no haptic feedback and with haptic feedback provided. Related result analysis of a user survey are discussed. The survey results indicate that the gloves provide compelling vibrotactile feedback.

The paper is organised as follows. A review of the related research work is given in Section II. In Section III, we focus on the description of the framework architecture. The two human subject studies are described in Section IV. In Section V, a user survey and the corresponding results are outlined. Finally, conclusions and future works are discussed in Section VI.

\section{RElated RESEARCH Work}

The miniaturisation of vibrotactile actuators have enabled researchers to develop compact and wearable haptic interfaces. Already in the 1990s, Cheng et al. [11] were some of the first researchers to use vibrotactile actuation in a glove. They used a $5 \mathrm{DT}^{2}$ sensing glove tracking the hand, combined with a Red Baron tracker tracking the wrist. Two vibrating actuators fixed to each fingertip conveyed ungrounded tactile feedback regarding contact interactions with virtual objects. Ten years later, Pabon et al. [12] presented an inexpensive haptic glove made of two goniometric sensors and three vibrotactile motors on each finger. Romano et al. [13] presented a vibrotactile glove able to convey ungrounded tactile feedback rendering slip sensations between the device and a virtual environment. Optical mouse sensors integrated in the glove are employed to sense the relative motion. This piece of information is provided to the wearer's fingertips through vibrotactile feedback. Krishna et al. [14] developed a haptic vibratory interface whose objective is to convey facial expressions to visually-impaired users. Three vibrotactile actuators on each fingertip convey this tactile information on human emotions. More recently, Muramatsu et al. [15], Galambos and Baranyi [16], and Foottit et al. [17] presented similar vibrotactile glove interfaces having one vibrotactile actuator per finger pad. The device developed by Muramatsu et al. also includes bend sensors to register the grasping pose, and that of Foottit et al. uses IMU and optical bend sensors to sense the hand and grasping pose, respectively. Vibrations at the fingertips have also been employed for outdoor navigation [18] and for telemanipulation [19].

With the objective of providing richer tactile sensations, researchers have also focused on non-vibrotactile solutions. A notable example is the set of tactile gloves presented by Prattichizzo et al. [20], [21] to display remote tactile experiences. An instrumented glove registers the interaction forces in the remote environment, and three 3-DoF cutaneous devices equipped with rigid platforms feeds those forces back to the human fingertips. However, although quite effective, non-vibrotactile solutions cannot provide the same compact design and level of wearability of the above-mentioned vibrotactile-only gloves. For these reasons, vibrotactile gloves are gaining great attention in the fields of virtual reality interaction and telerobotics.

To the best of our knowledge, a low-cost and open framework for a fully-immersive haptic, audio and visual experience is still missing. The main goal of this paper is to contribute towards the development of such a framework.

\section{FRAMEWORK ARCHITECTURE}

In this section, we describe the proposed framework architecture including software, hardware and multi-modal rendering strategies.

\section{A. Software Architecture}

The framework architecture is shown in Fig. 2-a. In the following, the key elements of the system are presented.

The principal component of the proposed framework is a pair of haptic gloves, shown in Fig. 2-b. Such gloves are fitted with precise shaft-less vibration motors embedded on the fingertips by using hook-and-loop fasteners. Using these actuators, it is possible to transfer distinctive haptic feedback patterns to the user to accurately simulate realistic finger collisions. The glove controller is implemented on an Arduino Uno board [22] and is based on the ATmega328 micro-controller. Arduino is an open-source electronics prototyping platform that employs flexible, easy-to-use hardware and software. Arduino provides a number of libraries to enable microcontroller programming through 


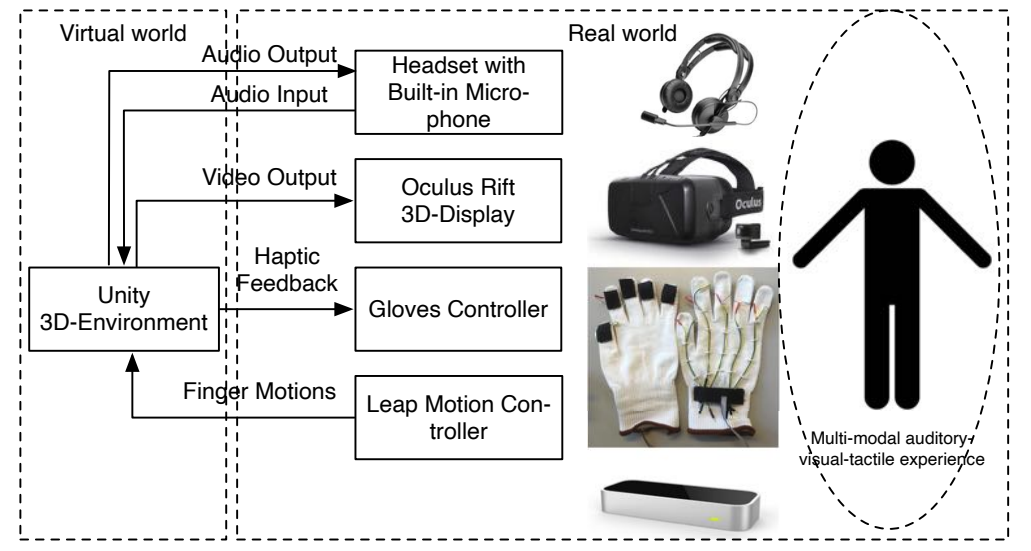

(a)

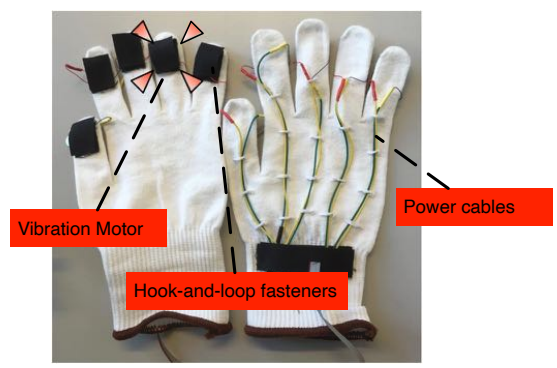

(b)

Fig. 2: The proposed framework: (a) the framework architecture, (b) the low-cost pair of haptic gloves.

rapid prototyping. As such, software development and in turn, hardware development, are simplified, thereby reducing the time needed to get the system running. The choice of using Arduino boards makes the system controller easy to maintain and allows new functionality to be introduced in the future. The newly designed gloves represent a significant advancement compared to similar COTS devices currently available on the market because they are easy to build by using only COTS components, they are based on open-source hardware and software, and are very durable and low-cost.

To sensorise the gloves and achieve tracking of the operator's hand and finger motions, a Leap Motion sensor [8] is adopted. The Leap Motion controller is a small portable Universal Serial Bus (USB) device designed to be mounted facing upwards on a physical desktop. Two monochromatic IR cameras and three infra-red LEDs are embedded in the device to monitor a roughly hemispherical area, to a distance of about 1 meter. The LEDs generate patternless IR light [23] and the cameras generate almost 200 frames per second of reflected data. The USB controller of the unit reads the sensor data into its own local memory and makes any necessary adjustments to the resolution. This data is then streamed via USB to the Leap Motion tracking software where 3D position data is synthesised by comparing the $2 \mathrm{D}$ frames generated by the two cameras with an overall average accuracy of 0.7 millimetres. The Leap Motion application programming interface (API) is then used for the integration with the visualisation environment. Even though the Leap Motion sensor is originally designed for hands-free motion tracking, it should be noted that the controller performs well even when wearing gloves (as shown in Section $\mathrm{V}$ from our experiments) or in combination with other wearables [21].

An Oculus Rift head-mounted 3D display [9] is used to provide the user with immersive visual feedback. An external bidirectional audio channel is supported by means of a headset with an integrated microphone to further enhance the user experience. In the same head-mounted set, all of these components are integrated.

The Unity cross-platform 3D-environment [10] is cho-

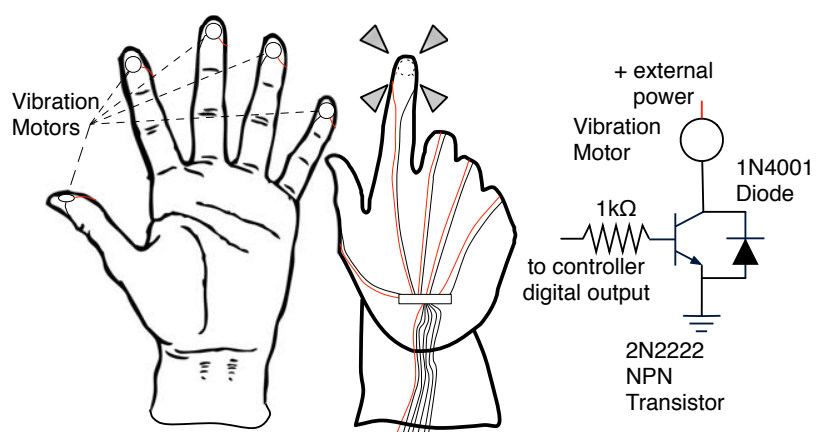

Fig. 3: Hardware schematics: one of the embedded vibration actuators and the corresponding control circuit.

sen as middleware for the integration of all framework elements. To achieve the desired integration, the correct application programming interface (API) is adopted for each component.

\section{B. Hardware schematics}

One of the embedded vibration actuators and the corresponding control circuit is shown in Fig. 3. This circuit consists of a $1 K \omega$ resistor, a $1 N 4001$ diode and a $2 N 2222$ $N P N$ transistor. The load uses $12 \mathrm{~V}$ and $300 \mathrm{~mA}$ of current.

\section{Multi-modal rendering strategies}

The tactile rendering system uses a force computation model, in which the amount of force displayed is proportional to the penetration depth or separation distance [24]. The $i$-th force $F_{i}$ is given by:

$$
F_{i}=k\left(t-d_{i}\right)-k_{v} v_{i},
$$

where $t$ is the distance tolerance for defining near contacts, $d_{i}$ is the distance value of the representative $i$-th contact, and $v_{i}$ is the $i$-th approaching velocity. While, $k$ and $k_{v}$ are stiffness and damping constants, respectively. The force is then rendered by adopting a Pulse Width Modulation (PWM) signal, where the duty cycle of the $i$-th vibration actuator, $D_{i}$, is proportional to the force to be rendered normalised between the specific actuator range, as depicted 


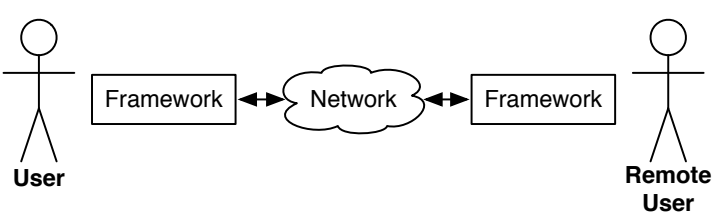

Fig. 4: A remote touch interaction between two users.

by the following equation:

$$
D_{i}=\frac{\alpha F_{i}-F_{\min }}{F_{\max }-F_{\min }}
$$

where $\alpha$ is a scaling factor. While, $F_{\min }$ and $F_{\max }$ are the minimum and maximum renderisable forces, respectively.

Similarly, auditory rendering is achieved by generating a sound feedback with a pitch that is proportional to the force to be rendered normalised between the specific frequency range. The $i$-th pitch frequency, $f_{i}$, is calculated according to the following equation:

$$
f_{i}=f_{\min } \frac{\beta\left(F_{i}-F_{\min }\right)\left(f_{\max }-f_{\min }\right)}{F_{\max }-F_{\min }},
$$

where $\beta$ is a scaling factor. While, $f_{\min }$ and $f_{\max }$ are the minimum and maximum renderisable frequencies, respectively.

Analogously, visual rendering is achieved for the collision points by generating a colour feedback with a wavelength that is proportional to the force to be rendered normalised between the specific wavelength range (visible spectrum). The $i$-th wavelength, $\lambda_{i}$, is calculated according to the following equation:

$$
\lambda_{i}=f_{\min } \frac{\gamma\left(F_{i}-F_{\min }\right)\left(\lambda_{\max }-\lambda_{\min }\right)}{F_{\max }-F_{\min }},
$$

where $\gamma$ is a scaling factor. While, $\lambda_{\min }$ and $\lambda_{\max }$ are the minimum and maximum renderisable wavelengths, respectively.

The multi-modal rendering is achieved by simultaneously combining auditory, visual and tactile rendering together.

The proposed multi-modal auditory-visual-tactile experience can be extended to a possible scenario for a remote tactile interaction as shown in Fig. 4. By implementing a network connection between two instances of the proposed framework.

\section{CAse Studies}

To demonstrate the potential of the proposed framework, two case studies are presented.

\section{A. Case Study 1}

A virtual scene is created in which the user wearing the gloves controls a quadcopter drone model. The objective is to navigate a scenario cluttered with obstacles, as shown in Fig. 5. Once the quadcopter is getting closer to an obstacle, the multi-modal feedback is rendered to the user. The user is able to control both the direction and the velocity of the drone using hand gestures. This first case

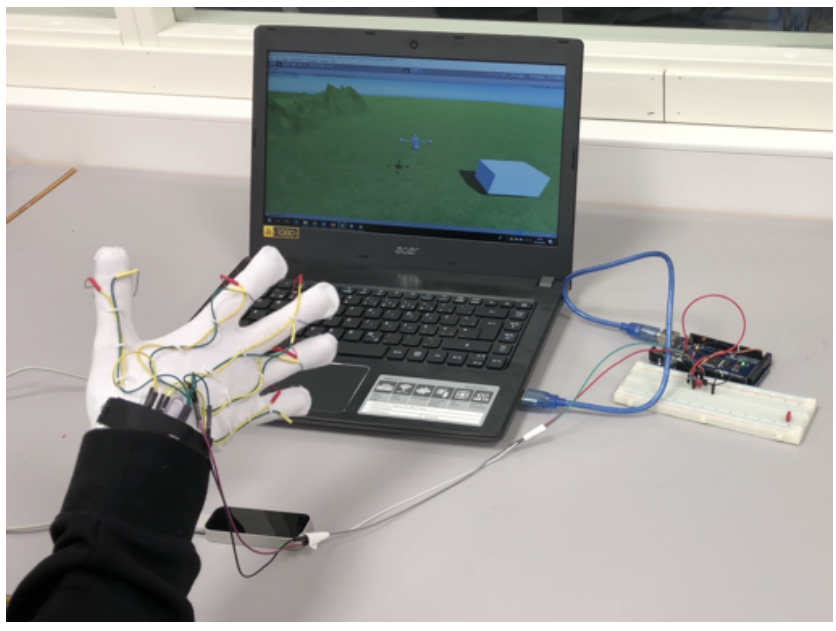

Fig. 5: Case Study 1: the user wearing the gloves controls a quadcopter drone model while navigating a scenario cluttered with obstacles in Unity.

study is meant to showcase from a qualitative point of view the variety of possible applications for the proposed framework.

\section{B. Case Study 2}

The user uses his index finger to interact with a virtual object, as shown in Fig. 6. The corresponding path tracking for the fingertip movement and accompanying motor activation pattern is monitored. This experiment is first performed by the user with only visual feedback enabled, without wearing the proposed haptic-glove (no tactile feedback is provided). The results are shown in Fig. 6-b. Successively, the same experiment is repeated by the user by wearing the proposed haptic-glove (tactile feedback is provided), as shown in Fig. 6-c. The user's movements appear to be more precise compared to the previous iteration of the experiment. This case study emphasise the importance of providing tactile feedback to enhance the user perception.

\section{SubJECTIVE QUESTIONNAIRE AND RESUlTS}

In addition to the two case studies, a subjective questionnaire was given to the user by adopting a 9-point Likert scale. The following questions were asked: (Q1) demographic information, such as age and sex; (Q2) how effective was the condition providing $*$ no* vibrotactile feedback?; (Q3) how effective was the condition providing vibrotactile feedback?; (Q4) did you have the feeling of performing better while using vibrotactile feedback or without? Data related to (Q1) are shown in Fig. 7-a-b. Results shown in Fig. 7-c-d, highlight that the condition providing vibrotactile feedback was rated with higher score by the users. The feeling of performing better while using vibrotactile feedback was unanimously stated by the users, as shown in Fig. 7-e. 


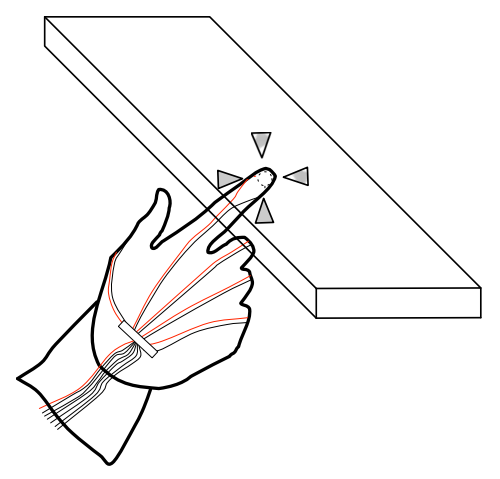

(a)

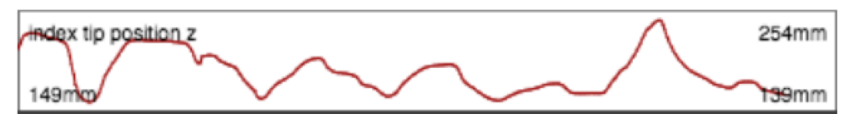

(b)

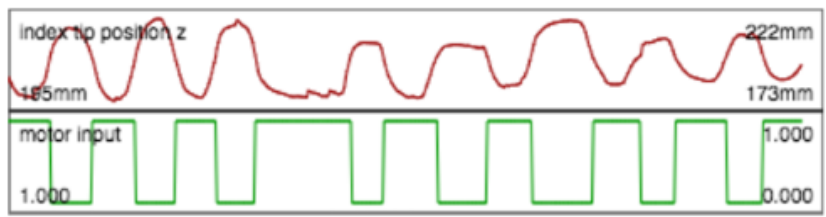

(c)

Fig. 6: Case Study 2: (a) the user interacts with a virtual object by using his index finger, (b) the corresponding path tracking for the fingertip height when the user is not wearing the proposed haptic-glove (no haptic feedback), (c) the corresponding path tracking for the fingertip height when the user is wearing the proposed haptic-glove (haptic feedback provided).

\section{CONCLUSIONS AND FUtURE WORK}

In [7], our research group previously introduced a lowcost framework for a fully immersive haptic, audio and visual experience. The architecture presented helps researchers to incorporate haptic capabilities to their systems conveniently. Distinctive functionalities can be easily achieved, including monitoring the hand and finger movements of the operator, detecting collisions between the virtual fingers of the operator and the virtual objects, measuring reaction forces in response to interactions and movements, and performing intuitive force feedback on the user. It is therefore possible to create a variety of local and remote applications.

In this work, the possibility of improving the robustness of the prototypes designed with the framework was considered. Accordingly, the concept of modularity is stressed even more than before concerning the control architecture and the software design. To explore the potential for possible applications, the usability of the framework is studied and evaluated. To demonstrate the effectiveness of the proposed system, two human subject studies were outlined. In the first case study, the the possibility for the user to control a quadcopter drone model while navigating

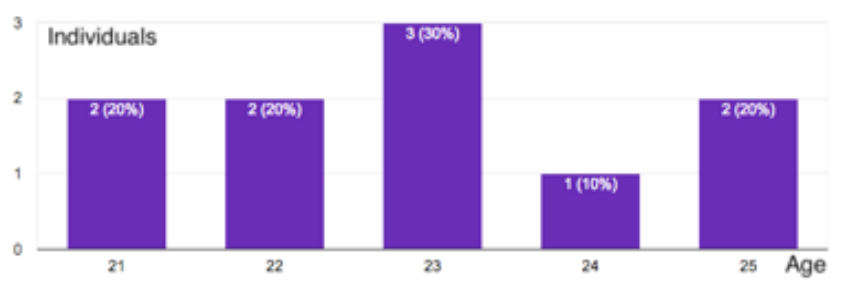

(a)

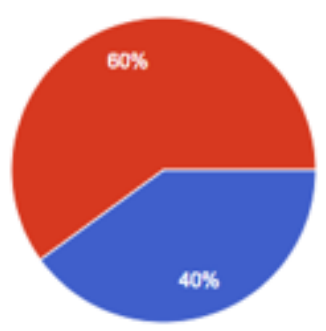

Sex: F

(b)

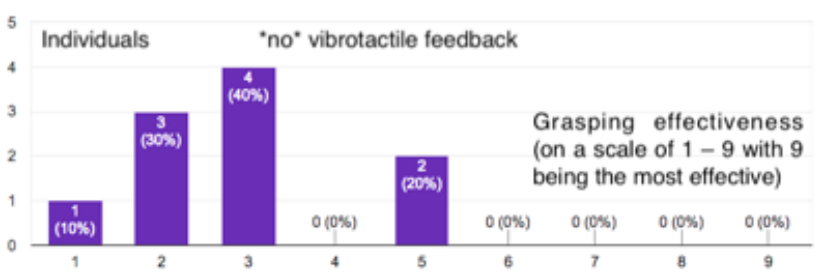

(c)

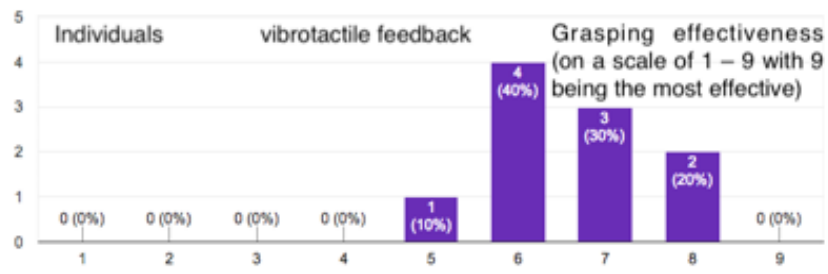

(d)

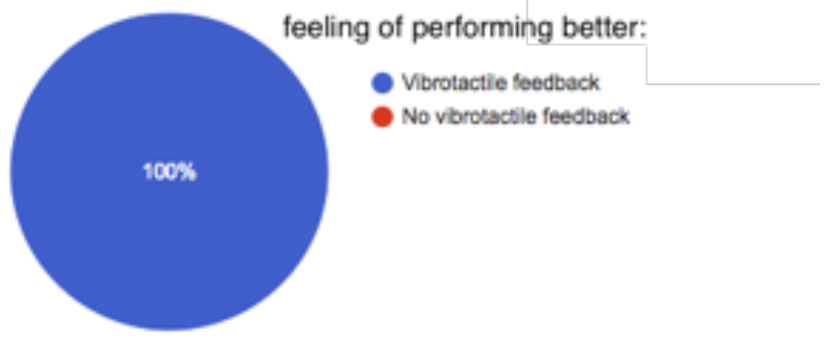

(e)

Fig. 7: Subjective questionnaire and results: (a) and (b) (Q1) demographic information, such as age and sex; (c) (Q2) how effective was the condition providing *no* vibrotactile feedback?; (d) (Q3) how effective was the condition providing vibrotactile feedback?; (e) (Q4) did you have the feeling of performing better while using vibrotactile feedback or without? 
a scenario cluttered with obstacles was demonstrated. The user was able to use hand gestures to control both the direction as well as the velocity of the drone. Haptic feedback was provided to the users when the drone was approaching obstacles in order to avoid collisions during navigation. In the second case study, the user used his index finger to interact with a virtual object. The corresponding path tracking for the fingertip movement and accompanying motor activation pattern demonstrated the importance of providing tactile feedback to enhance the user perception.

Related result analysis of user surveys were outlined. The set of results collect indicates that the gloves can be used to provide compelling vibrotactile feedback in an effective way.

This type of interfaces show a great potential in several fields. One of the most promising application scenario is gaming. This opportunity has been already exploited by a few startup companies. Immerz (USA) raised USD 183,000 on Kickstarter for their "KOR-FX" gaming vest. It converts audio signals coming from the game into vibrotactile haptic stimuli that allow the wearer to feel in-game events such as explosions and punches. A similar experience is promised by the "Feedback jacket" by Haptika (PK), the full-body suit "Teslasuit" by Tesla Studios (UK), and the "3RD Space Vest" by TN Games (USA). However, no vibrotactile glove has been commercialised yet to the best of our knowledge. The proposed system demonstrates that a low-cost solution is feasible using only commercial off-the-shelf (COTS) components.

In the future, the possibility of adding new functionalities to the proposed framework may be considered. For instance, the possibility of exposing haptic devices physically connected to remote computers, available through a computer network, as if they were directly connected to the running host. It should be noted that all the new features can be logically layered without modifying the presented library.

\section{ACKNOWLEDGMENTS}

This work is supported by the Top Research Centre Mechatronics, University of Agder (UiA), Jon Lilletuns vei 9, 4879, Grimstad, Norway. The authors gratefully acknowledge the contribution of Steven Bos, Tuan Minh Hua, Fredrik Kåsin and Kristen Haave.

\section{REFERENCES}

[1] M.-L. Bourguet, "Designing and prototyping multimodal commands." in Interact, vol. 3. Citeseer, 2003, pp. 717-720.

[2] A. Tirmizi, C. Pacchierotti, and D. Prattichizzo, "On the role of cutaneous force in teleoperation: subtracting kinesthesia from complete haptic feedback," in Proc. of the IEEE World Haptics Conference (WHC), 2013, pp. 371-376.

[3] M. Aggravi, F. Pausé, P. R. Giordano, and C. Pacchierotti, "Design and evaluation of a wearable haptic device for skin stretch, pressure, and vibrotactile stimuli," IEEE Robotics and Automation Letters, vol. 3, no. 3, pp. 2166-2173, 2018.

[4] M. Sreelakshmi and T. Subash, "Haptic technology: A comprehensive review on its applications and future prospects," Materials Today: Proceedings, vol. 4, no. 2, pp. 4182-4187, 2017.
[5] C. Pacchierotti, S. Sinclair, M. Solazzi, A. Frisoli, V. Hayward, and D. Prattichizzo, "Wearable haptic systems for the fingertip and the hand: taxonomy, review, and perspectives," IEEE transactions on haptics, vol. 10, no. 4, pp. 580-600, 2017.

[6] F. Sanfilippo, P. B. Weustink, and K. Y. Pettersen, "A coupling library for the force dimension haptic devices and the 20-sim modelling and simulation environment," in Proc. of the 41st Annual Conference of the IEEE Industrial Electronics Society (IECON2015), Yokohama, Japan. Submitted, 2015.

[7] F. Sanfilippo, L. I. Hatledal, and K. Pettersen, "A fully-immersive hapto-audio-visual framework for remote touch," in Proc. of the 11th IEEE International Conference on Innovations in Information Technology (IIT'15), Dubai, United Arab Emirates, 2015.

[8] Leap Motion Inc. (2020, February) "The Leap Motion Controller". [Online]. Available: http://www.leapmotion.com/

[9] Oculus VR. (2020, February) "Oculus Rift Development Kit 2". [Online]. Available: http://www.oculus.com/

[10] Unity Technologies. (2020, February) "Unity3D”. [Online]. Available: http://unity3d.com/

[11] L.-T. Cheng, R. Kazman, and J. Robinson, "Vibrotactile feedback in delicate virtual reality operations," in Proc. ACM Int. Conf. on Multimedia, 1997, pp. 243-251.

[12] S. Pabon, E. Sotgiu, R. Leonardi, C. Brancolini, O. PortilloRodriguez, A. Frisoli, and M. Bergamasco, "A data-glove with vibro-tactile stimulators for virtual social interaction and rehabilitation," in Proc. Annual Int. Workshop on Presence, 2007.

[13] J. M. Romano, S. R. Gray, N. T. Jacobs, and K. J. Kuchenbecke, "Toward tactilely transparent gloves: Collocated slip sensing and vibrotactile actuation," in Proc. World Haptics, 2009, pp. 279-284.

[14] S. Krishna, S. Bala, T. McDaniel, S. McGuire, and S. Panchanathan, "Vibroglove: an assistive technology aid for conveying facial expressions," in Proc. Int. Conf. on Human Factors in Computing Systems, 2010, pp. 3637-3642.

[15] Y. Muramatsu, M. Niitsuma, and T. Thomessen, "Perception of tactile sensation using vibrotactile glove interface," in Proc. IEEE Int. Conf. on Cognitive Infocommunications, 2012, pp. 621-626.

[16] P. Galambos and P. Baranyi, "Vibrotactile force feedback for telemanipulation: Concept and applications," in Proc. IEEE Int. Conf. on Cognitive Infocommunications, 2011, pp. 1-6.

[17] J. Foottit, D. Brown, S. Marks, and A. Connor, "Development of a wearable haptic game interface," EAI Endorsed Transactions on Creative Technologies, vol. 16, no. 6, 42016.

[18] I. Dabran, S. Shalev, A. Shiber, and O. Raitzes, "A smart outdoor navigation system for visually impaired people," in Proc. of the 12th ACM International Conference on Systems and Storage, 2019, pp. $184-184$.

[19] J. H. Low, W. W. Lee, P. M. Khin, N. V. Thakor, S. L. Kukreja, H. L. Ren, and C. H. Yeow, "Hybrid tele-manipulation system using a sensorized 3-d-printed soft robotic gripper and a soft fabric-based haptic glove," IEEE Robotics and Automation Letters, vol. 2, no. 2, pp. 880-887, 2017.

[20] D. Prattichizzo, F. Chinello, C. Pacchierotti, and K. Minamizawa, "Remotouch: A system for remote touch experience," in Proc. IEEE International Symposium on Robots and Human Interactive Communications, 2010, pp. 676-679.

[21] S. Scheggi, L. Meli, C. Pacchierotti, and D. Prattichizzo, "Touch the virtual reality: using the leap motion controller for hand tracking and wearable tactile devices for immersive haptic rendering," in ACM SIGGRAPH 2015 Posters. ACM, 2015, p. 31.

[22] Arduino. (2020, February) Arduino, an open-source electronics prototyping platform. [Online]. Available: http://arduino.cc/

[23] F. Weichert, D. Bachmann, B. Rudak, and D. Fisseler, "Analysis of the accuracy and robustness of the leap motion controller," Sensors, vol. 13, no. 5, pp. 6380-6393, 2013.

[24] Y. J. Kim, M. A. Otaduy, M. C. Lin, and D. Manocha, "Sixdegree-of-freedom haptic rendering using incremental and localized computations," Presence: Teleoperators \& Virtual Environments, vol. 12 , no. 3, pp. 277-295, 2003. 